\title{
Exploring exoplanetary systems beyond 1AU with WFIRST
}

\author{
Matthew T. Penny ${ }^{1}$ and B. Scott Gaudi ${ }^{1}$ \\ ${ }^{1}$ Department of Astronomy, The Ohio State University, 140 W. 18th Ave., Columbus, OH, \\ USA 43210 \\ email: penny@astronomy.ohio-state.edu, gaudi@astronomy.ohio-state.edu
}

\begin{abstract}
The Wide Field InfraRed Survey Telescope (WFIRST) was the top ranked large space mission of the New Worlds, New Horizons Decadal Survey, and is currently under active study by NASA. Its primary instrument will be a large-format high-resolution near-infrared imager and slitless spectrometer. A primary goal of WFIRST will be to perform a high-cadence microlensing survey of the Galactic bulge to search for low-mass exoplanets beyond the ice line. We highlight some of the expected results of the WFIRST exoplanet survey. For example, the survey will probe the abundance of Earth-mass planets from less than 1 AU outwards, including free-floating planets. In its peak sensitivity range of $\sim 2-5$ AU, WFIRST will be sensitive to planets with masses lower than Mercury, and even down to the mass of Ganymede. Overall, WFIRST is expected to detect several thousand bound planets, in addition to several thousand free-floating planets. WFIRST will complete the exoplanet census begun by Kepler, enabling an unprecedented understanding of planetary systems and their formation.
\end{abstract}

Keywords. planets and satellites: detection, planetary systems, gravitational lensing: micro, space vehicles, telescopes

WFIRST will be a wide-field $\left(\sim 0.3 \mathrm{deg}^{2}\right)$, diffraction-limited $(\sim 0.16-0.29$ arcsec $)$, nearinfrared survey spacecraft using either a 1.3-m telescope (Green et al. 2012) or a donated 2.4-m telescope (Spergel et al. 2013). A primary mission goal, the microlensing survey will run for 432 days over five years to detect hundreds of free-floating planets and thousands of cold, bound planets down to the mass of solar system moons. Deep, high-resolution imaging over a long time baseline will allow planet hosts to be imaged after they have separated from the source stars they lens, enabling the measurement of host and planet masses for most hosts.

Using the MaB $\mu \mathrm{LS}$ simulator (pronounced may-buls, Penny et al. 2013) we have simulated the WFIRST microlensing survey. Figure 1 shows two representations of the expected yield of WFIRST in relation to current exoplanet discoveries. Plotted as a function of planet mass and orbital semimajor axis, the blue shaded region shows the region in which the 2.4-m version of WFIRST can be expected to find more than 3 planets if there is one such planet per star, with the dashed line showing the equivalent mass sensitivity for free-floating planets. The red shaded region shows the nominal sensitivity of an extended Kepler mission to transiting planets. Blue points show the expected distribution of WFIRST detections if cold exoplanets follow the mass function measured by Cassan et al. (2012) and conservatively extrapolated to masses below $5 M_{\oplus}$ (see Penny et al. 2013 for details). Black points show currently known exoplanets while red points show Kepler planet candidates. Solar system planets, and three of their largest satellites Ganymede, Titan and the Moon - are denoted by colourful symbols. The figure also highlights the capabilities of the WFIRST microlensing survey and shows an example of low-mass bound and free-floating planet detections. 


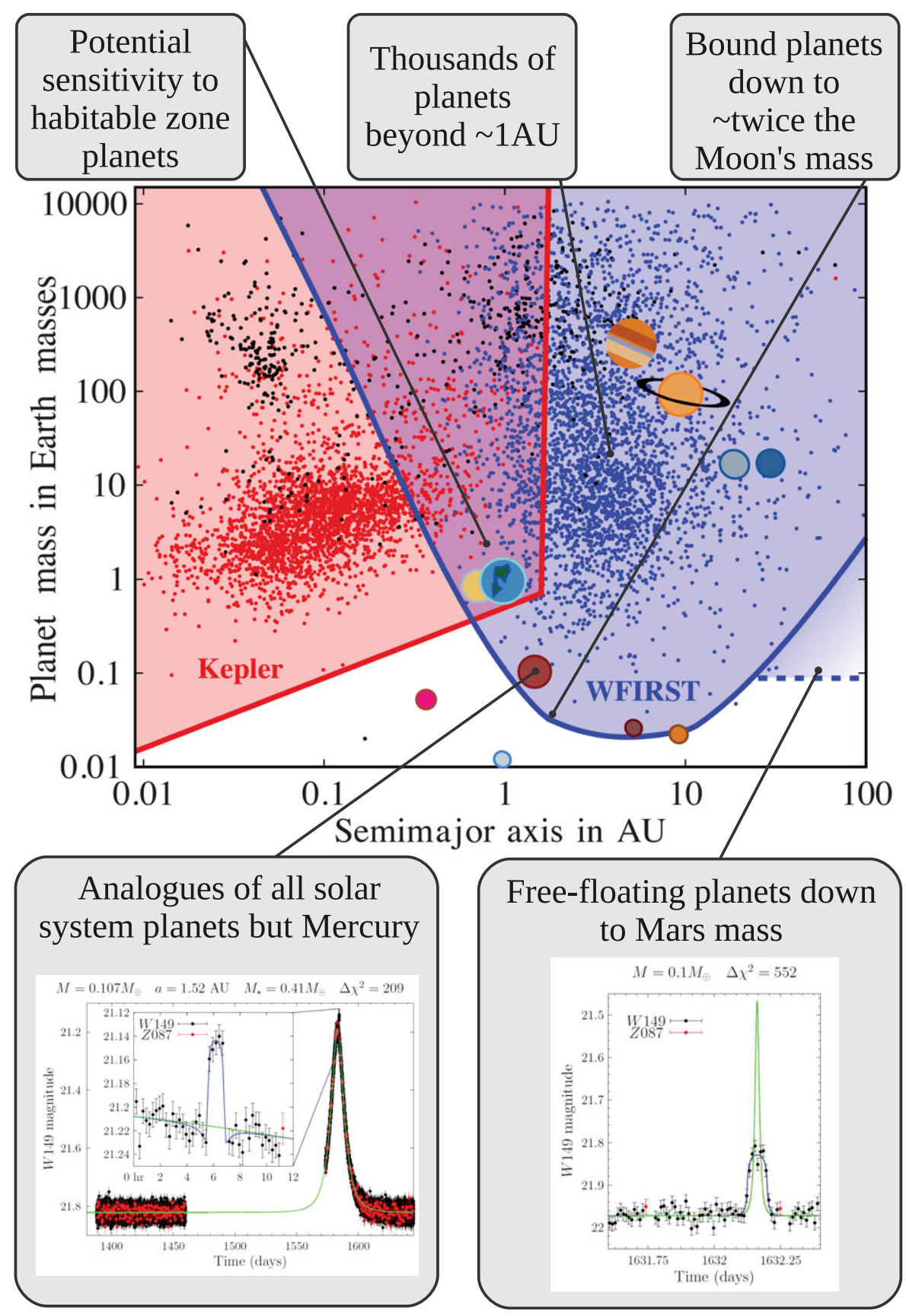

Figure 1. Predictions for the exoplanet microlensing survey of WFIRST and examples of simulated detections of low-mass bound and free-floating planets.

\section{References}

Green, J., et al. 2012, arXiv 1208.4012

Spergel, D., et al. 2013, arXiv 1305.5422

Penny, M. T., et al. 2013, MNRAS, 434, 2

Cassan, A., et al. 2012, Nature 481, 167 